\section{الآثار التفسية للخبرات الحياتية الصادمة: معاناة مهملة}

Psychological effects of traumatic life experiences: neglected sufferance

Prof. Dr. Samer J. Rudwan ". د سامر جميل رضوان

اضطر ابات "الثدة التالية للصدمة" هي نوع من الاضطر ابات النفسية الناجمة عن تعرض الإنسان لأحداث و أزمات ذا طبيعة محزنة أو مخيفة، كالمصائب و الكوارث و الرزايا، سو اء طبيعية، كالز لازل و الفيضانات و الحر ائق، أو من صنع الإنسان، كالحروب والاستغلال الجنسي، إلخ. وعلى الرغم من أن تعرض الإنسان لبعض أو كثثر من الضغوطات الحياتية أمر طبيعي، ولا مفر منه، إلا أن بعض الأفراد أقل قدرة على التحمل من البعض الآخر فيعانون مما يُسمى باضطر ابات الخبرة الحياتية الصادمة التي سنتطرق إلى بعضها هنا، وكيفية مواجهتها بأقل التكاليف.

\title{
Abstract
}

Post-traumatic stress disorder is a type of mental sufferance that can develop in people who are exposed to traumatic events, caused by humans such as wars and sexual exploitation, or by life natural events, such as death, incurable illnesses, earthquakes, fires, etc. Although the exposure to stressful life events is part of the human life, some individuals are more vulnerable than others and, consequently, they suffer more than others. We will address these questions and provide some guidelines on how to face them and reduce their harmful effects to the least possible.

\section{الأزمات النفسية في سياق تاريخي وعربي}

لعل الثعب العربي-عبر تاريخه- من أكثر الشعوب تعرضاً للأزمات والصدمات النفية الناجمة عن الحروب والكوارث التي صنعتها يد الإنسان. فمنذ فجر التاريخ، وحتى يومنا الراهن، شهدت بلادنا من الكوارث والمصائب والصدمات ما لا يعد ولا يحصى، سواء من حيث النوع أو الكم. صدمات اخترقت وتخترق بنية الإنسان المادية والنفسية، لتمزقها وتتركها مشوهة، وتمتد آثارها لسنوات عديدة، وربما طوال العمر، مخلّفة ورائها الحزن

\footnotetext{
*Prof. Dr. Samer J. Rudwan, Department of Education \& Cultural Studies, College of Arts \& Sciences, University of Nizwa, Oman. Email:srudwan@hotmail.com : الدكتور سامر جميل رضو ان، جامعة نزوى، سلطة عمان، البريد الإلكتروني:
} 
واليأس والاستسلام والخوف من المجهول والإحساس بعقدة الضحية الفاقدة للثقة بكل ما حولها، وتتوقع الخطر في كل لحظة وتشكك بنفسها وبالآخرين، وتتقوقع حول نفسها، وتجعل من أفعالها مجرد ردود أفعال طائشة تعيد من

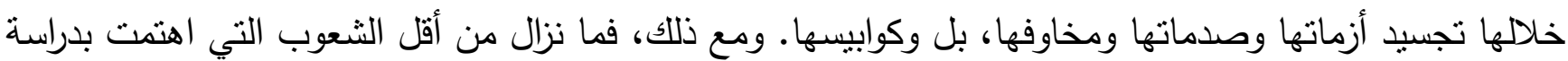
الآثار النفسية الناجمة الكوارث والأزمات قياساً على الكم الهائل من أزماتتا النفسية. سنحاول في هذا المقال إلقاء الضوء على هذا النوع من الاضطرابات من وجهة النظر النفسية، زيادة في فهمها ودعوة للاهتمام بها وعدم تجاهلها.

اضطرابات "الثدة التالية للصدمة"، والتي نفضل تسميتها "اضطرابات الضغط التالية للصدمة"، هي التسمية التي تطلقها منظمة الصحة العالمية، والجمعية النفسية الأمريكية على ذلك النوع من الاضطرابات النفسية الناجمة

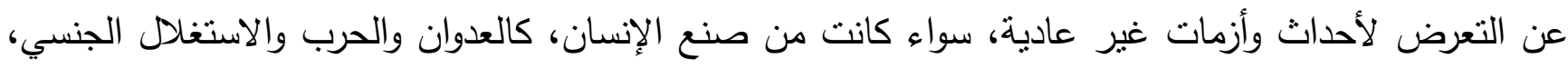

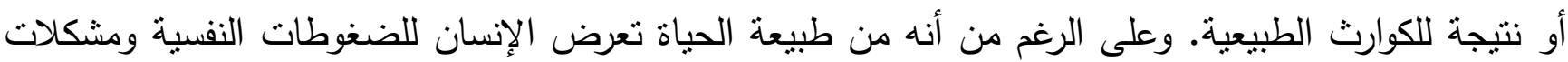
سوء التكيف، فإن المقصود بالأزمة هنا هو الخبرة التي تكون خارج نطاق الخبرة الحياتية العادية أو المألوفة

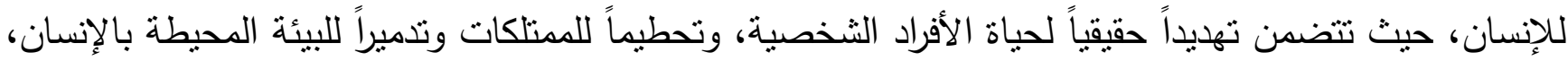
مع ما يصاحب ذللك من مشاهد عنف ودمار ، سواء كان الثخص نفسه ضحية للعنف والدمار والغبن الجنسي، أو مشاهداً لمناظر العنف والدمار تلك. وتتنتمل الخبرات الصادمة وفق التصنيفات العالمية على الخبرات التالية: - أحروب بكافة أشكالها.

- الاعتداء الجسدي على الثخص ( كالاغتصاب والغبن الجنسي، والضرب، والنهب والسلب).

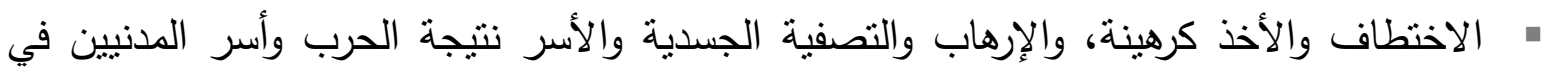

$$
\text { معسكرات الاعتقال. }
$$

- الكوارث الطبيعية أو الناتجة عن تأثثر الإنسان نفسه.

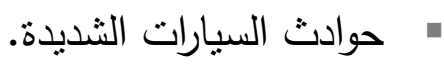
- ت تشخيص مرض خطير مهدد للحياة. - الأحداث التي لا يكون الثخص نفسه ضحية لها، وإنما مراقباً أو مشاهداً، كمشاهدة إصابة شديدة أو مشاهدة حالة موت غير طبيعي نتيجة حادث أو عمل إجرامي أو قتالي أو نتيجة لكارثة طبيعية، أو المشاهدة غير المتوقعة للجسد المقتول أو لجزء منه تعتبر أيضاً خبرات صدادمة.

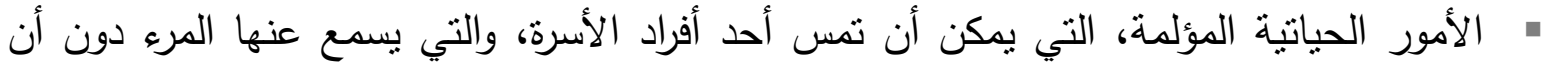
يراها هي أحداث حياتية صادمة أيضاً للكثيرين. 
- الخبرات الجنسية عند الأطفال غير المتتاسبة مع مستوى نضجهم هي خبرات صادمة جنسياً لهم، حتى وإن لم تترافق هذه الخبرات بالتهديد أو العنف.

ومن الناحية الكمية، يمكن القول إن الأحداث الحياتية الصادمة الناجمة عن فعل الإنسان أثند وقعاً وتأثثراً، من بأن

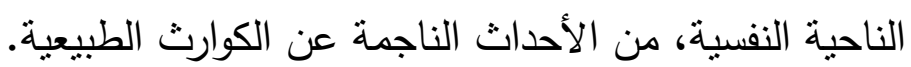

ولا تقتصر الآتار الناجمة عن الحروب أو الحوادث أو الكوارث الطبيعية أو العنف (الجنائي) أو عن التعذيب على

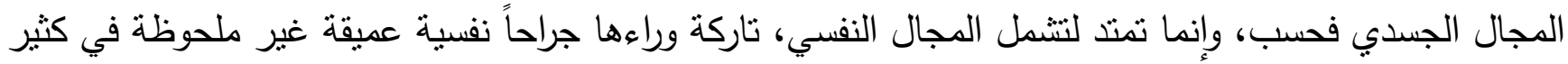
من الأحيان، تؤثر سلباً على من تعرضوا إلى شكل من أنثال الأحداث الحياتية الصادمة. وغالباً ما يُهنت بالإصابات الجسدية الناجمة عن الأحداث الحياتية الصادمة، ومعالجتها بسرعة كي تشفى، ويُتجاهل في الوقت التهات نفسه الإصابات النفسية الناجمة عن ذللك، فتكبت في أغوار المصابين، وتهمل من قبل المتخصصين.

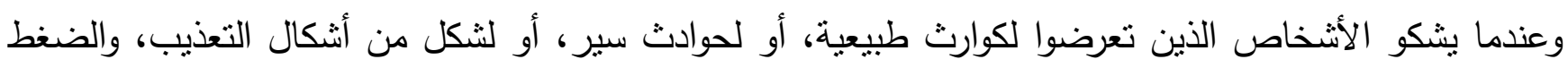

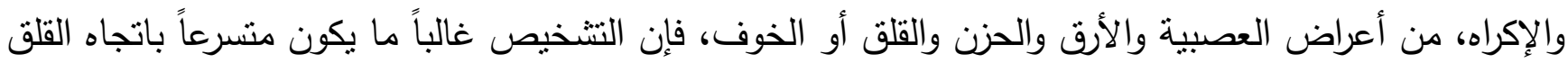

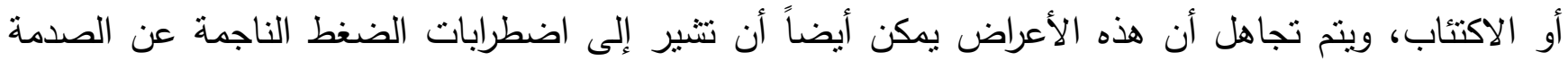

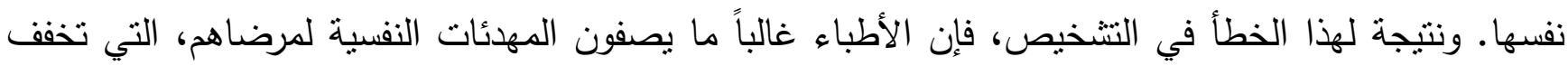

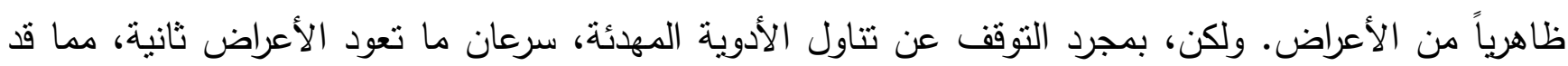
يتسبب في وقوع المرضى في حلقة التعود أو الإدمان على العقاقير النفسية.

\section{أعراض اضطرابات الضغط التالية للصدمة}

تختلف العواقب النفسية للخبرات الصادمة باختلاف الأفراد، غير أن هناك مجموعة من الأعراض المشتركة

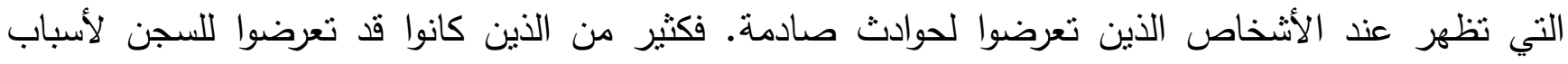

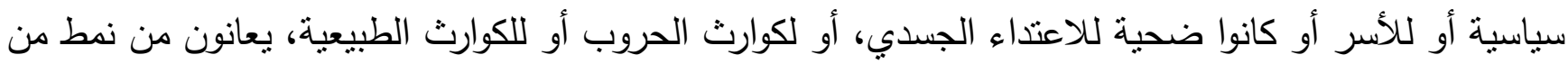
الأعراض يتجلى باضطرابات النوم (الأرق)، والكوابيس المرعبة، والانعزال الاجتماعي، وفقدان الاهتمام الاكتئابي،

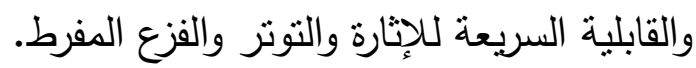
ويمكن تقسيم الأعراض المتتوعة لاضطرابات الضغط الناجمة عن صدمة حياتية إلى ثلاثة فئات رئيسية هي:

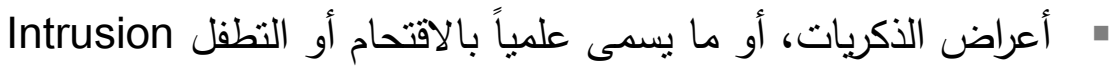

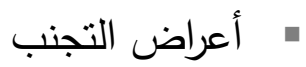

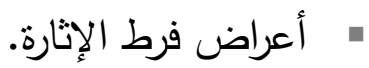


أعراض الذكريات

من أكثر ما يميز صورة الاضطراب هو الذكريات المتكررة والمرهقة للخبرة الصادمة، والتي تقتحم ذهن الثخص المعني بدون أب مناسبة. والأسوأ من ذلك أن كثير من المعنيين يعانون من مشاعر الذنب، لأنهم عاشوا هم أو نجوا في حين أن الآخرين قتلوا، أو لأنهم اضطروا للقيام بتصرفات معينة من أجل إنقاذ حياتهم، ويحمّلون

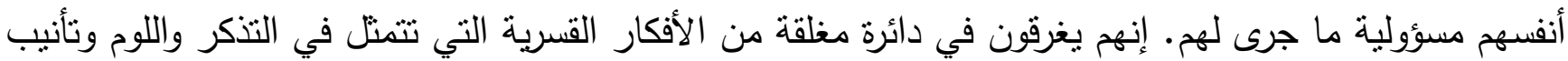
الذات، وقد لا بستطيعون التخلص من هذه الثثلاثية المرهقة، لأن الذكريات المؤلمة قد تباغتهم بشكل متكرر . إنها

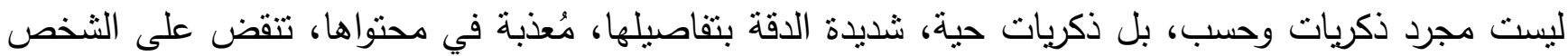

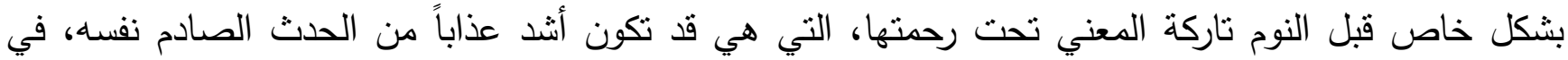
كثير من الحالات.

ولا يقتصر الأمر على الذكريات الواعية، بل قد يمند ليشتمل الأحلام أيضاً، والتي تتكرر فيها مشاهد مفرطة الدقة

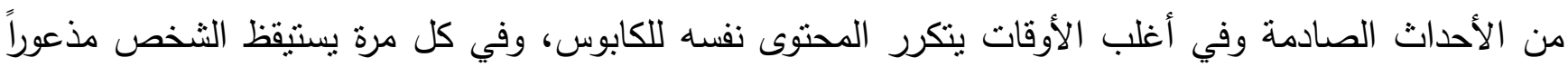
والعرق يتصبب منه خوفاً وهلعاً. كما وقد لا يتوقف الأمر على مجرد تكرار خبرات الحدث الصادم، أو الأحداث الصادمة في الذكريات والأحلام فحسب، بل يمكن أن يقوم الثخص المصدوم، وبشكل مفاجئ بالتصرف - أو أن يشعر - وكأنه يعيش من جديد الحدث الصادم بتفاصبله. وترتبط أعراض التذكر مع مشاعر قويه تضع المعنيين في حالة صدمة ومعاناة نفسية قاسية، تبرز بشكل خاص عندما يواجهون مواقف شبيهة تذكرهم بالحدث الصادم الذي عاشوه.

\section{أعراض التجنب}

غالباً ما يحاول المعنيون، بشكل شعوري أو لا شعوري، كبت الأفكار وتجنب المواقف التي تذكرهم بما عاشوه من صدمة، وذللك من أجل حماية الذات من الضغوطات الناجمة عن أعراض التذكر . ويتجلى ذلك بصورة واضحة من خلال الانسحاب الاجتماعي. فنجدهم يرفضون نلبية الدعوات، ويتخلون عن واجباتهم وهواياتهم عموماً، حيث يلاحظ وجود انخفاض واضح في الاهتمام بالمشاركة في النشاطات الاجتماعية المهمة، بعد خبرة حدث صادم

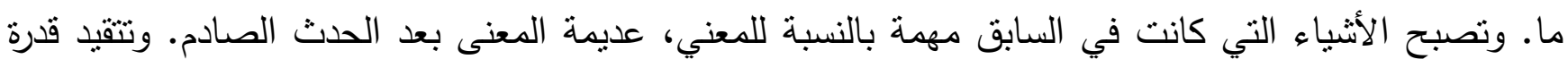

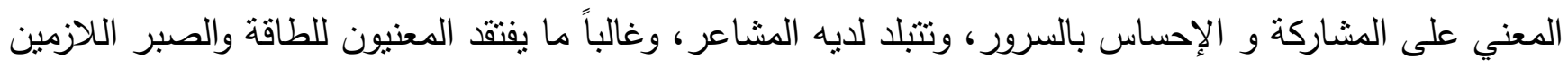
لبناء المخططات المستقلية، حيث يعاش المستقبل هنا بأنه غامض أو محجوب (ويتجلى ذلك في حالة الأطفال

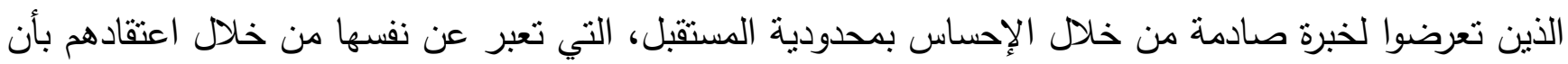

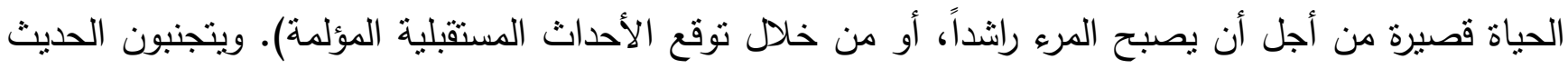


عن خبراتهم المؤلمة، وبشكل خاص عن بعض التفاصيل، أو على الرغم من أن الكوابيس غالباً ما تكون على شكل

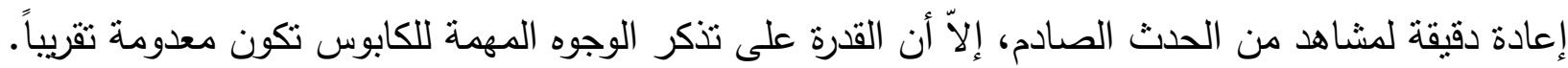

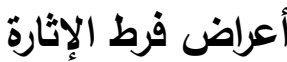

إن من تُعذب الذكريات أفكاره ومشاعره يتشكل لديه فرط إثارة داخلية، يمكن أن تقود إلى ردود فعل الفزع،

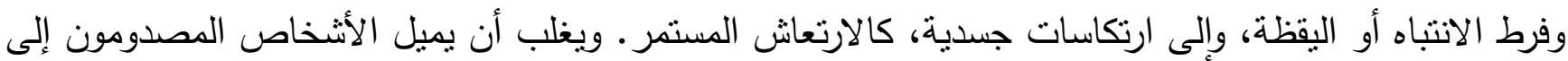

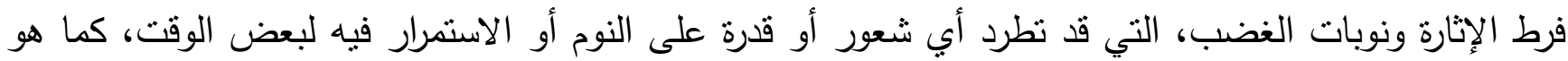

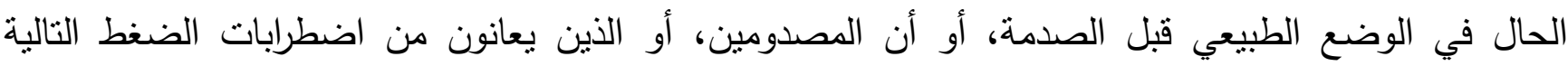

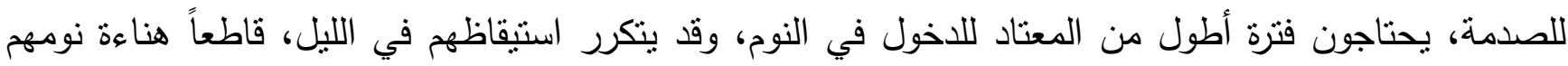

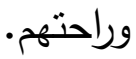

\section{المشكلات الناجمة عن معاناة حدث صادم}

إلى جانب اضطرابات الضغوط التالية للصدمة، يمكن ملاحظة أو حدوث اضطرابات نفسية أخرى، كالقلق والاضطرابات الاكتئابية. وغالباً ما يقود فرط الإثارة الجسدية إلى أوجاع وشكاوى جسدية. ولأن كثير من المعنيين

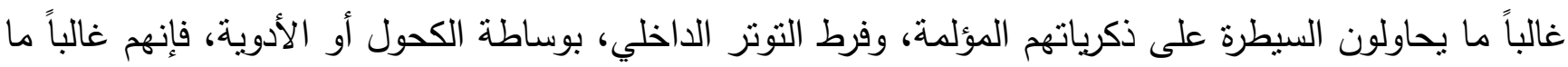
ينزلقون أيضاً في دوامة أخرى لا تقل خطورة وهي، فرط استهلاك الكحول أو سوء استخدام الأدوية.

ردود أفعال طبيعية على أحداث شاذة

يمكن لاضطرابات الضغط التالية للصدمة - وبشكل خاص عندما يتعلق الأمر بخبرة غير منوقعة كلية ومرهقة بشكل منطرف - أن تظهر عند الناس الذين كانوا قبل ذلك أصحاء كلية، أي حتى عند الذين لم يكونوا قبل الصدمة يمتلكون دلائل على وجود اضطرابات نفسية أخرى، أو استعداداً مسبقاً للإصابة بشكل من أثنكال

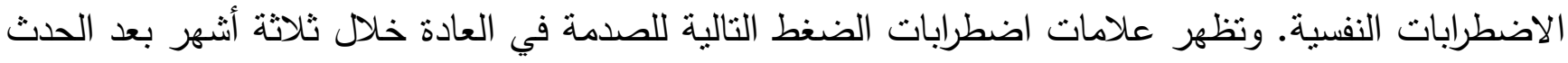
الصادم. ويمكن هنا أن يأخذ مجرى الاضطراب الثنكل الحاد أو المزمن. فإذا ما استمرت الأعراض لفترة تلتجاوز

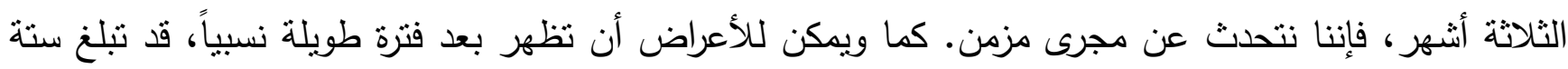

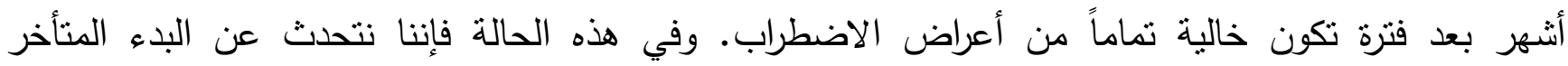
ل اضطراب.

\section{المعالجة النفسية لاضطرابات الضغط التالية للصدمة}


غالباً ما يُعيق تجنب المصدوم الحديث عن كل ما يُّكّره بتجربته الصادمة محاولة البحث عن المساعدة المتخصصة، الأمر الذي يقود إلى أن يأخذ الاضطراب الثكل المزمن. وعلى الرغم من ذللك، فقد أثنتت دراسات

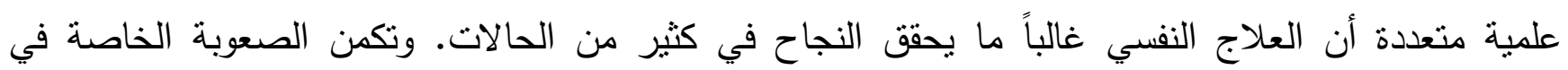
المعالجة النفسية لاضطرابات الضغط التالية للصدمة في خطر قطع المعالجة من قبل المعنيين، ذلك أن توجيه

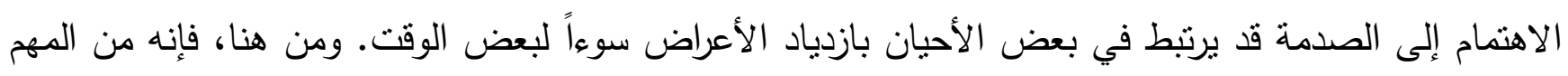

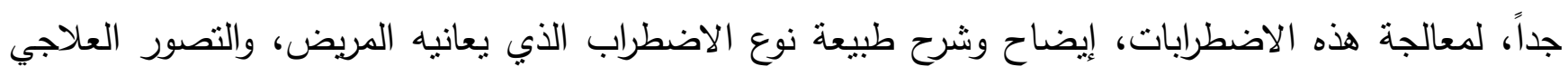
ومجرى المعالجة.

ويطلب من المرضى في مجرى المعالجة، قدر الإمكان، تصور مواقف الخبرة الصادمة بكل أفكارهم ومشاعرهم

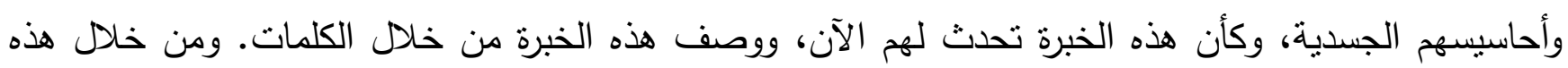

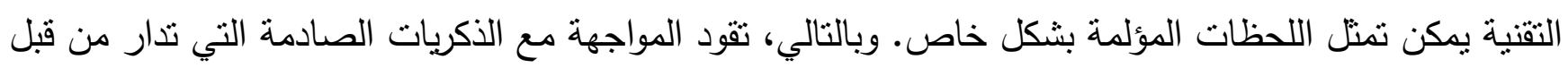
المعالج النفسي بطريقة مهنية مضبوطة إلى تراجع الأعراض الناجمة عن الصدمة. ويمكن اختيار أساليب علاجية مختلفة أو التوليف بين أساليب علاجية متتوعة أو متكاملة، وذلك حسب طبيعة الحالة الفردية، وطبيعة الخبرة الصادمة التي تعرض لها الثخص. ومن الطرق التي تستخدم هنا، على سبيل المثال، المحادثات العلاجية التي لتئه تهدف إلى التغلب على دور الضحية، واليأس وفقدان السيطرة أو لعب الأدوار (السيكودراما) أو المواجهة التدريجية لهنية

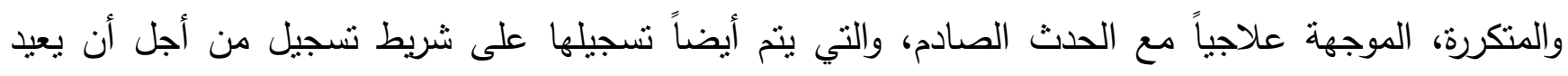

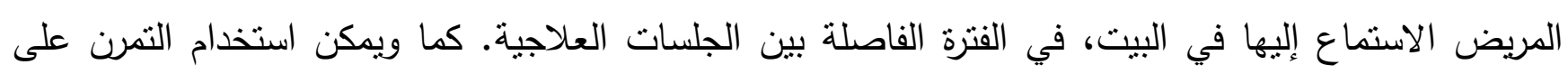

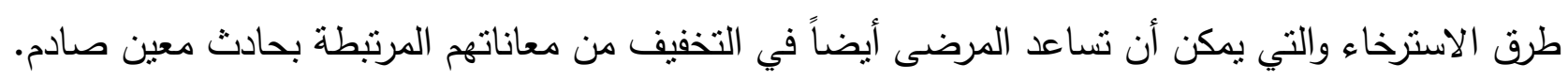

